\title{
Simulación de Mecanismos
}

\section{Mechanism Simulation}

\author{
JIMÉNEZ-RABIELA, Homero†**, VÁZQUEZ-GONZÁLEZ, Benjamín, RAMÍREZ-CRUZ, José Luis \\ y GARCÍA-SEGURA, Pedro
}

\begin{abstract}
Universidad Autónoma Metropolitana, Unidad Azcapotzalco, División de Ciencias Básicas e Ingeniería, Departamento de Energía
\end{abstract}

ID $1^{\mathrm{er}}$ Autor: Homero, Jiménez-Rabiela / Researcher ID Thomson: S-2299-2018, CVU CONACYT ID: 123386

ID $1^{\text {er }}$ Coautor: Benjamín, Vázquez-González / ORC ID: 0000-0002-9030-5662, Researcher ID Thomson: S-2417-2018, CVU CONACYT ID: 25749

ID $2^{\text {do }}$ Coautor: José Luis, Ramírez-Cruz / ORC ID: 0000-0003-0762-2630, Researcher ID Thomson: G-3405-2019, CVU CONACYT ID: 921268

ID $3^{\text {er }}$ Coautor: Pedro, García-Segura / ORC ID: 0000-0003-4947-084X, Researcher ID Thomson: S-2360-2018, CVU CONACYT ID: 371233

DOI: $10.35429 / \mathrm{JCS} .2019 .7 .3 .20 .27$

Recibido: 12 de Enero, 2019; Aceptado 30 de Marzo, 2019

\section{Resumen}

Objetivos: En este trabajo se muestra diferentes mecanismos simulados virtualmente, su objetivo es facilitar el análisis cualitativo y cuantitativo de su cinemática; permitiendo determinar grados de libertad, relación de tiempos, ventaja mecánica, movilidad, posiciones, velocidades y aceleraciones. Tal objetivo se logra simulándolos para diferentes posiciones usando discretización y temporización variable. Metodología: Consta de archivos de mecanismos con los archivos correspondientes de eslabones y hojas electrónicas de cálculo. El alumno debe modificar las restricciones dimensionales, geométricas y cinemáticas; eliminarlas e instalar nuevas; para observar el comportamiento del mecanismo como un sistema o de los eslabones como partes integrantes. Contribución: Simplifica el proceso cognitivo de la cinemática de los mecanismos. Facilita la comprensión del enfoque de Euler y de Lagrange. Permite comprender y evaluar las posiciones, velocidades y aceleraciones; absolutas, relativas y aparentes. Los archivos de mecanismos y eslabones son virtuales, tridimensionales y permiten su animación. Las hojas electrónicas de cálculo controlan las restricciones tanto de los mecanismos como de sus eslabones.

Simulación, Mecanismos, Análisis

\begin{abstract}
Objectives: In this work we show different simulated mechanisms virtually, their objective is to facilitate the qualitative and quantitative analysis of their kinematics; allowing to determine degrees of freedom, relation of times, mechanical advantage, mobility, positions, speeds and accelerations. Such an objective is achieved by simulating them for different positions using discretization and variable timing. Methodology: It consists of files of mechanisms with the corresponding files of links and electronic spreadsheets. The student must modify the dimensional, geometric and kinematic restrictions; remove them and install new ones; to observe the behavior of the mechanism as a system or of the links as integral parts. Contribution: Simplify the cognitive process of the kinematics of the mechanisms. It facilitates the understanding of the Euler and the Lagrange approaches. It allows to understand and evaluate the positions, speeds and accelerations; absolute, relative and apparent. The files of mechanisms and links are virtual, threedimensional and allow their animation. Electronic spreadsheets control the restrictions of both the mechanisms and their links.
\end{abstract}

Simulation, Mechanisms, Analysis

Citación: JIMÉNEZ-RABIELA, Homero, VÁZQUEZ-GONZÁLEZ, Benjamín, RAMÍREZ-CRUZ, José Luis y GARCÍASEGURA, Pedro. Simulación de Mecanismos. Revista de Simulación Computacional. 2019. 3-7: 20-27

\footnotetext{
* Correspondencia al Autor: (hjr@azc.uam.mx)

$\dagger$ Investigador contribuyendo como primer autor.
} 


\section{Introducción}

Analizar los diferentes mecanismos desde el punto de vista de la cinemática de los mismos es un proceso complejo. Requiere la aplicación de enfoques de Euler y de Lagrange. Adicionalmente requiere evaluar posiciones, velocidades y aceleraciones absolutas, relativas y aparentes. Los conceptos anteriores resultan de difícil comprensión por parte de los alumnos.

En este trabajo se generaron archivos gráficos de ensamble con sus correspondientes archivos gráficos de eslabones; adicionalmente, archivos de hojas electrónicas de cálculo para controlar tanto los archivos de ensamble como los de eslabones. Es importante en tanto que facilita al alumno el análisis cualitativo y cuantitativo de la cinemática de mecanismos.

Tales análisis se facilitan con la simulación de los mecanismos para diferentes posiciones con discretización y temporización variable. Las características grafo-analíticas agregan valor al proceso; haciendo posible su empleo en aulas, laboratorios, talleres, industrias y centros de investigación; su importancia durante este siglo ha propiciado la utilización de herramientas de simulación para diversos fines. En noviembre de 2005 Becker M. C. et al argumentaron algunas de sus ventajas apoyándose en un estudio de casos de la industria automotriz europea.

En 2007 Schuler J. et al presentaron una novedosa herramienta de modeladomanufactura asistida por computadora para mecanismos esféricos. En 2012 Alvarez J. C. \& $\mathrm{Su} \mathrm{H}$. J. presentaron un entorno virtual intuitivo para soportar el diseño conceptual de mecanismos. También en 2012, López P. R. et al mostraron el desarrollo de una herramienta para simular el movimiento del mecanismo manivelabiela-corredera.

En 2015, Robson N. \& Ghosh S. presentaron resultados del diseño geométrico de mecanismos planos basados en manipulación virtual. Respecto al uso de modelos físicos, los virtuales permiten discretizaciones tan finas como lo desee el usuario; adicionalmente permiten congelar aquellas imágenes de posición del mecanismo que puedan ser de su interés.
También y no menos importante, es la simulación de los mecanismos que resultan de la cadena cinemática, al considerar sus inversiones, esto permite al alumno visualizar a una mayor profundidad los conceptos de posiciones, velocidades $y$ aceleraciones relativas $y$ aparentes, normalmente de difícil comprensión por parte de los alumnos.

El carácter virtual, tridimensional y paramétrico permiten simulaciones de los mecanismos con diferentes restricciones dimensionales geométricas y cinemáticas.

Analizar los diferentes mecanismos (RRRR, RRRP, RRPR, levas, engranes,...) con teorías particulares para cada uno de ellos agrega complejidad, innecesaria, al proceso cognitivo. Usando las longitudes cinemáticas de los eslabones es posible analizar los diferentes mecanismos con una teoría única, aquella correspondiente al mecanismo de cuatro barras RRRR, simplificando el proceso.

En la introducción se hace una reseña histórica de la aplicación de la realidad virtual al tema de mecanismos. En nomenclatura se definen parámetros y acrónimos. En ensambles generados se indican los mecanismos obtenidos. En mecanismo RRRR, se indican las convenciones necesarias para permitir que la teoría aplicable a este mecanismo se pueda aplicar a cualquier otro. En Mecanismos RRRR particulares se muestran ejemplos de mecanismos y su visualización como cuatro barras RRRR. En Otros mecanismos simulados se incluyen algunos en los que las posiciones, velocidades y aceleraciones aparentes pueden entenderse mejor con la ayuda de las simulaciones. En Ley de Grashof se corrobora su validez para todo mecanismo. En los últimos apartados del artículo, se incluye resultados, agradecimiento, conclusiones y referencias.

\section{Nomenclatura}

La longitud cinemática del eslabón i se desinó con $L_{i}$. Con $\theta_{i}$ se designó al ángulo del eslabón correspondiente, con respecto al eje $\mathrm{X}$ positivo. El centro instantáneo de velocidades de los eslabones i y j se designó con $O_{i j}$. Con $\omega_{i}$ se designó a las velocidades angulares. Se designó a las aceleraciones angulares con $\alpha_{i}$. Para el ángulo de la cuña se usó $\beta$. Se usó DC para la distancia entre centros. 
Los subíndices variando de 1 a 4 . Se uso la letra $\mathrm{M}$ como acrónimo de mecanismo, C como acrónimo de coseno, e como acrónimo de excentricidad.

\section{Ensambles generados}

Algunos son mecanismos: Acoplamiento de Oldham, Contacto de rodadura, Contacto directo, Eslabonamiento equivalente excéntrica, Eslabonamiento equivalente RP permanente, Eslabonamiento RP temporal, Eslabonamiento equivalente RR permanente, Eslabonamiento RR temporal, Junta de Hooke, Leva de cuña y seguidor con rodillo, Leva de placa con seguidor de cara plana, Mecanismo de cuatro barras RRRP, Mecanismo RRRR posiciones velocidades y aceleraciones, Mecanismo de sujeción, Mecanismo en 3D esférico, Mecanismo en 3D RCCR, Mecanismo en 3D RESP, Mecanismo en 3D RSSR, Planetario, Planetario fijos engrane anillo y armadura planetaria, Planetario fijo el sol, Robot 1R 3P, Robot 2R 1P 1C, Robot 4R 1P, Tranvía elíptico, Yugo escocés. Otros son pares cinemáticos: Cilíndrico, Cinco GDL, Cuatro GDL U, Cuatro GDL V, Esférico, Helicoidal, Plano, Prismático, Revoluta. Todos pueden ser simulados en condiciones diferentes a criterio del usuario.

\section{Mecanismo RRRR}

El mecanismo RRRR, mostrado en la Fig. 1, es el caso general; el eslabón 1 es el café, el 2 es el rojo, el 3 es el azul y el 4 es el verde.

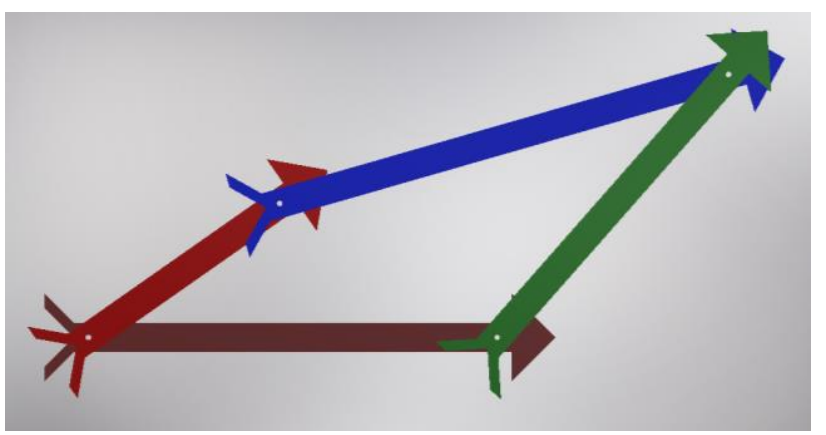

Figura 1 Mecanismo RRRR.

Fuente: Elaboración Propia

Todo mecanismo puede ser visualizado como tal. Las longitudes cinemáticas de los eslabones son: Eslabón 1, distancia de $O_{12}$ a $O_{14}$; eslabón 2, distancia de $O_{12}$ a $O_{23}$; eslabón 3 , distancia de $O_{23}$ a $O_{34}$; eslabón 4, distancia de $O_{14}$ a $O_{34}$. Las imágenes de posiciones, velocidades y aceleraciones, correspondientes al mecanismo de la Fig. 1, se muestran en la Fig. 2.

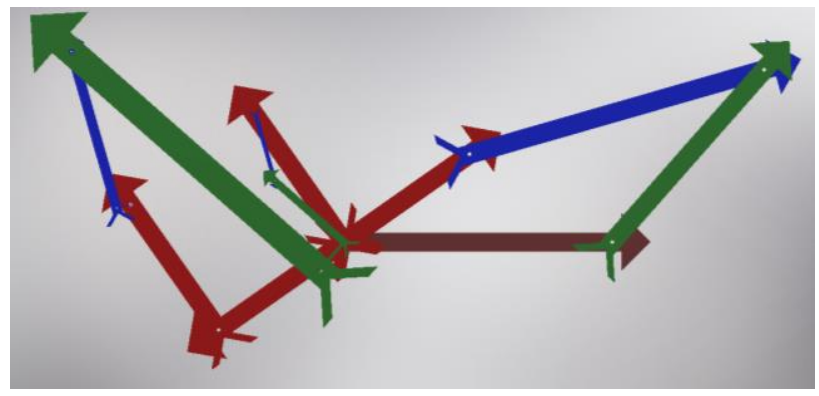

Figura 2 Polígonos RRRR

Fuente: Elaboración Propia

En tanto que parametrizado es posible obtener las posiciones, velocidades y aceleración para diferentes mecanismos RRRR. En las Figs. 3 y 4 , se muestran para dos de ellos.

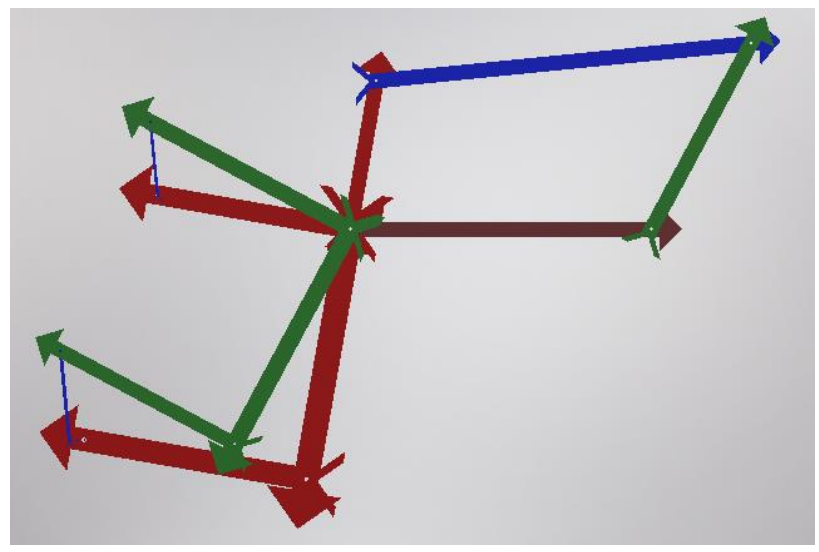

Figura 3 Mecanismo general Fuente: Elaboración Propia

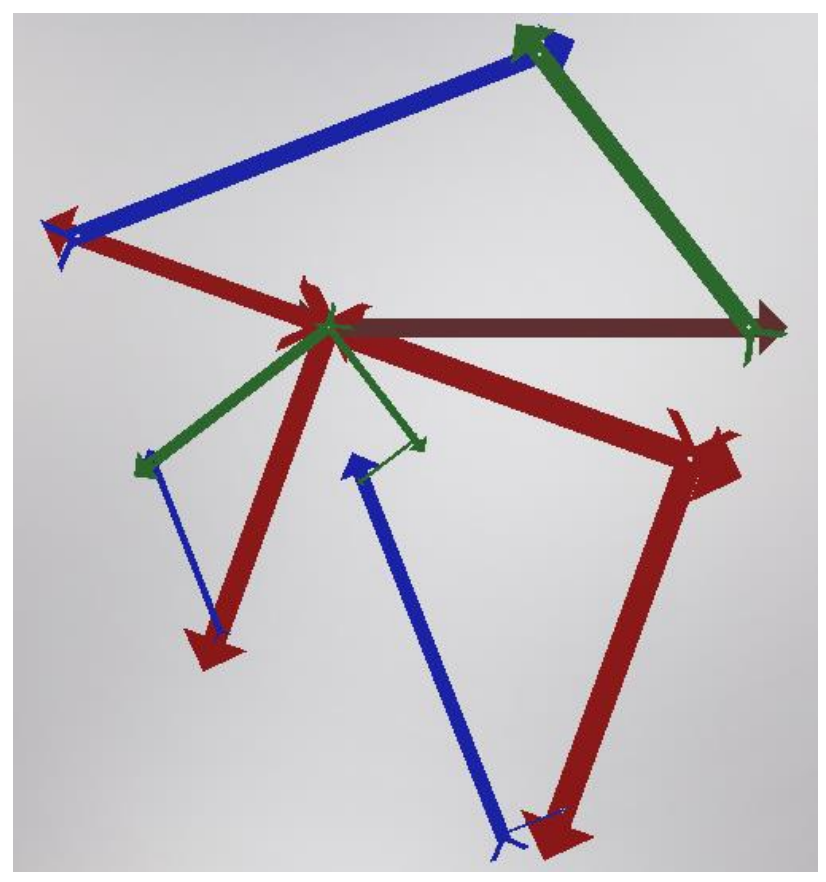

Figura 4 Mecanismo general Fuente: Elaboración Propia

Nótese que tanto las longitudes como los anchos de las imágenes de velocidades y aceleraciones son función de $\omega_{2}$ y $\alpha_{2}$. 
En la Tabla 1 se muestran los parámetros usados para los polígonos de las Figs. 2 a 4.

\begin{tabular}{|l|l|l|l|}
\hline & Fig. 2 & \multicolumn{1}{l}{ Fig. 3 } & Fig. 4 \\
\hline$L_{1}$ & 7 & 10 & 11 \\
\hline$L_{2}$ & 4 & 5 & 7 \\
\hline$L_{3}$ & 8 & 12.5 & 13 \\
\hline$L_{4}$ & 6 & 7 & 9 \\
\hline$\theta_{2}$ & 35 & 80 & 160 \\
\hline$\omega_{2}$ & 1 & 1.3 & 1.2 \\
\hline$\alpha_{2}$ & 1 & 1.5 & 1.4 \\
\hline
\end{tabular}

Tabla 1 Polígonos RRRR

Fuente: Elaboración Propia

\section{Mecanismos RRRR particulares}

Algunos mecanismos se visualizan como mecanismos RRRR en las siguientes Figs. En las Figs. 5 y 6 se muestran dos discos; ambos girando excéntricamente y sus centros geométricos definen al eslabón acoplador. Durante el movimiento del mecanismo las longitudes cinemáticas de los eslabones equivalentes permanecen invariantes. $\mathrm{Si}$ los radios de los discos son iguales y su excentricidad también, el mecanismo equivalente será de paralelogramo.

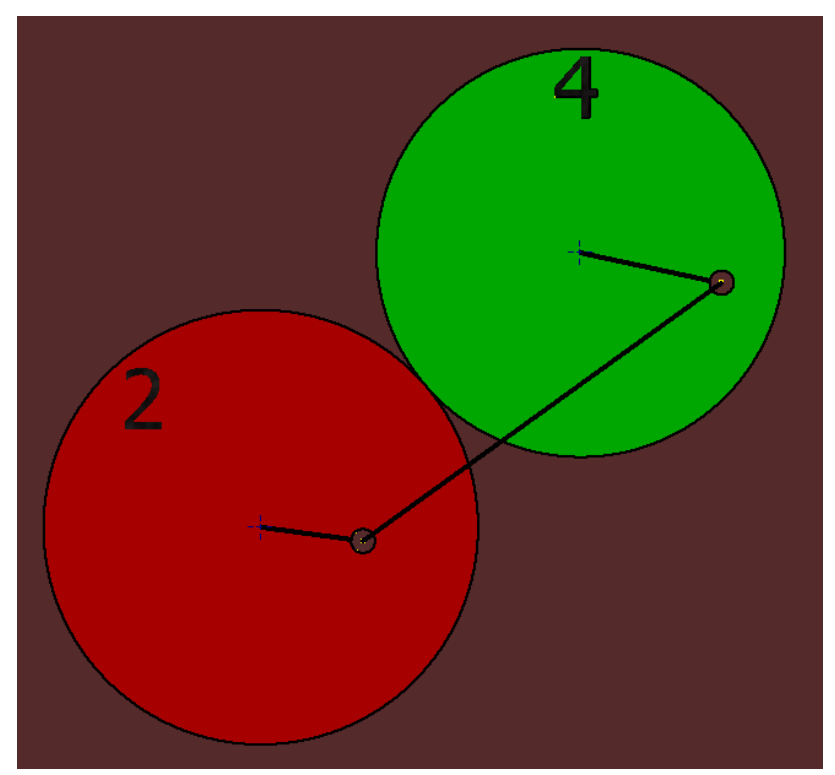

Figura 5 Leva y seguidor girando

Fuente: Elaboración Propia

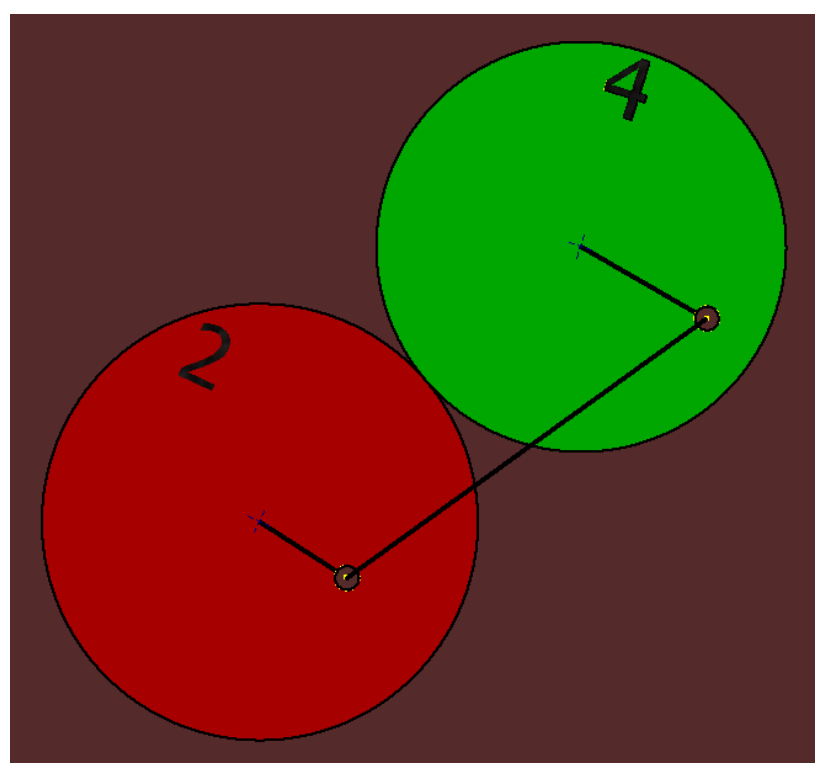

Figura 6 Leva y seguidor girando Fuente: Elaboración Propia

El tranvía elíptico visualizado como mecanismo RRRR se muestra en las Figs. 7 y 8. La longitud cinemática de los eslabones 2 y 4 es infinita. La del eslabón 1 es.

$L_{1}=\infty+L_{3} \cos \theta_{3}$

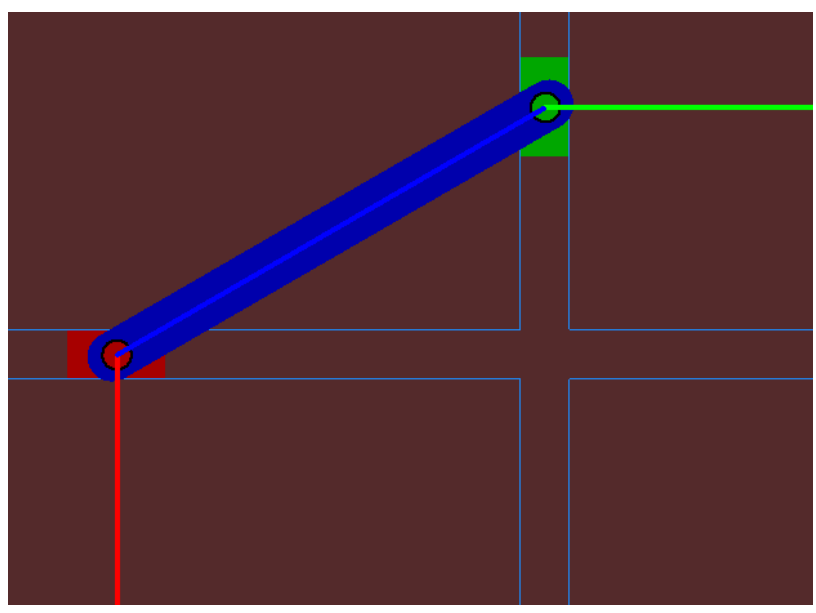

Figura 7 Tranvía elíptico

Fuente: Elaboración Propia 


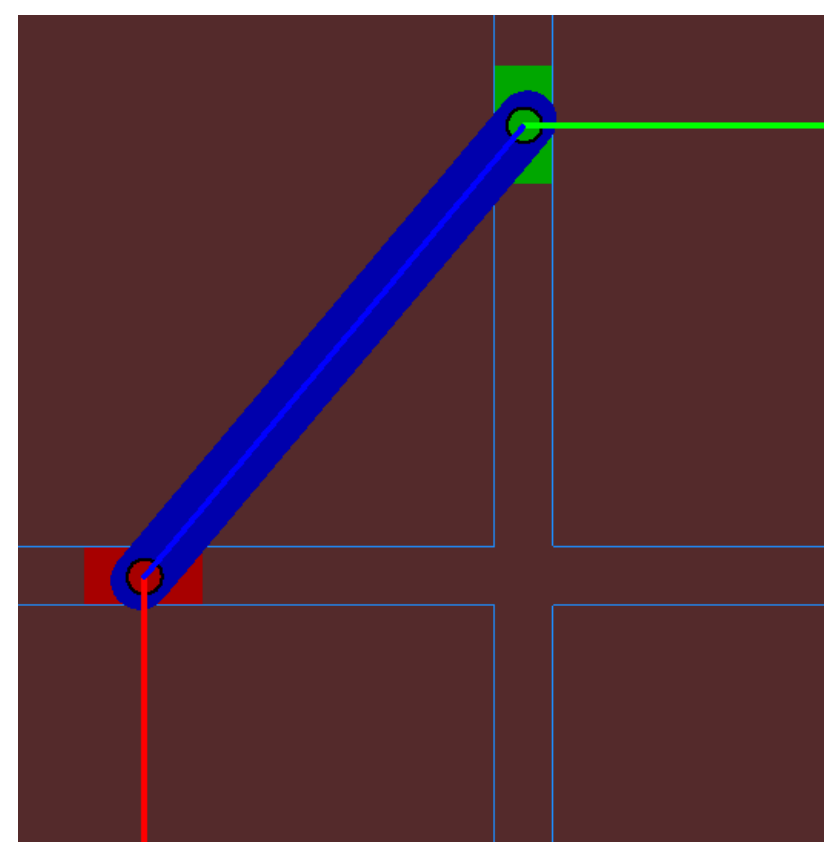

Figura 8 Tranvía elíptico

Fuente: Elaboración Propia

Un mecanismo en el que existe un par cinemático de rodadura se visualiza como un mecanismo RRRR en la Fig. 9. Las longitudes cinemáticas de todos los eslabones son finitas, la de los eslabones 1, 2 y 3 es invariante, la del eslabón 4 es variable.

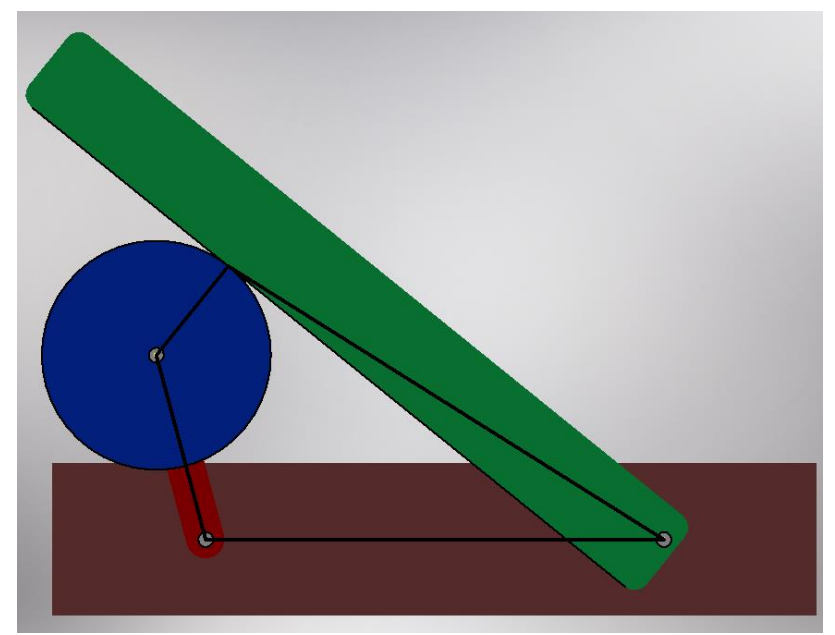

Figura 9 Contacto de rodadura Fuente: Elaboración Propia

Un mecanismo particular de leva y seguidor en el que existe un par cinemático de rodadura se visualiza como un mecanismo RRRR en la Fig. 10. Sólo la longitud cinemática del eslabón 3 es finita. Nótese que está fijo el eslabón opuesto al más corto, lo que implica que, si cumple Grashof, el mecanismo debe ser oscilador - oscilador.

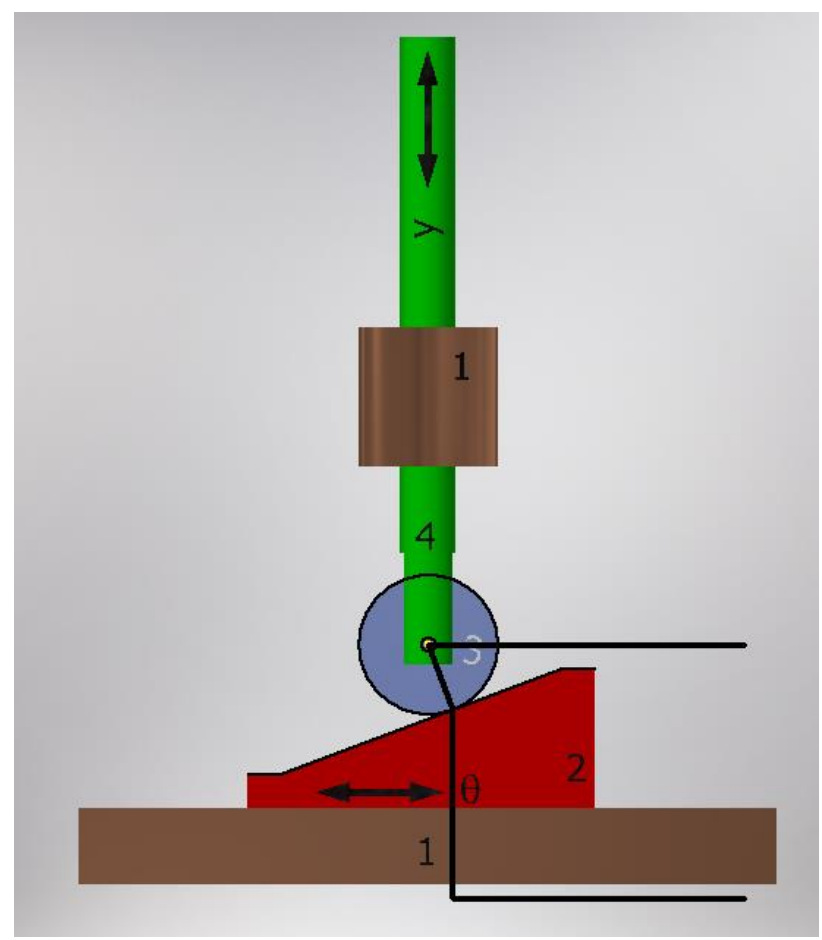

Figura 10 Leva y seguidor Fuente: Elaboración Propia

Para un mecanismo de engranes visualizado como mecanismo RRRR, mostrado en la Fig. 11, las longitudes cinemáticas de los eslabones 2 y 4 corresponden al radio de la circunferencia base respectiva.

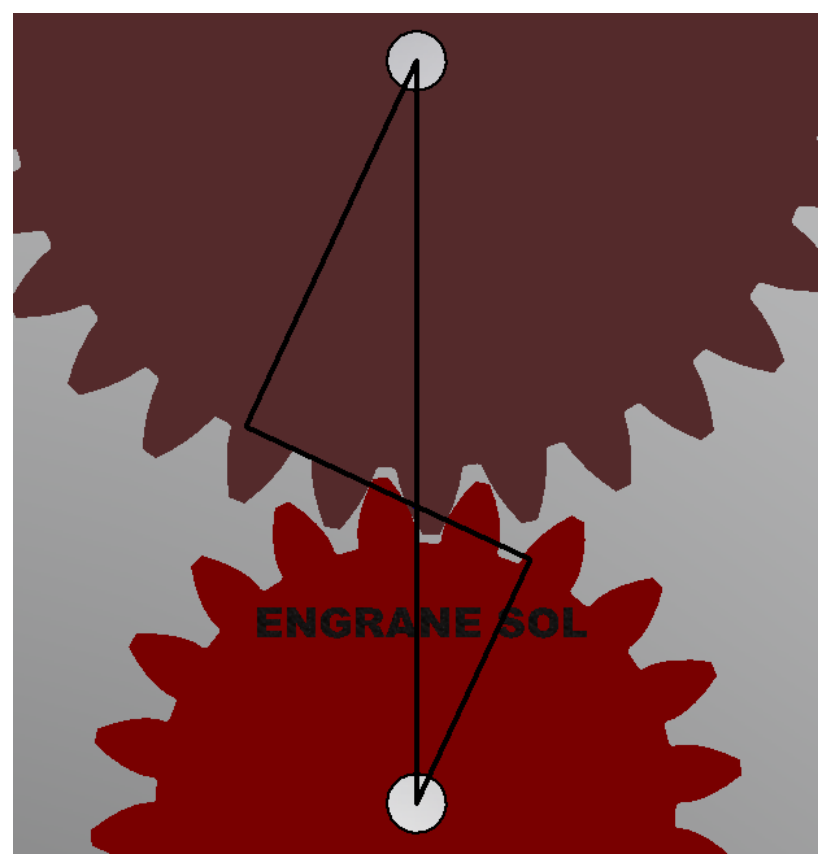

Figura 11 Leva y seguidor girando Fuente: Elaboración Propia

Las longitudes cinemáticas de los eslabones 2 y 3 en un mecanismo manivelabiela-corredera descentrado son finitas, ver Fig. 12. La del eslabón 1 es infinita y la del eslabón 4 es. 
$L_{4}=\infty+e$

(2)

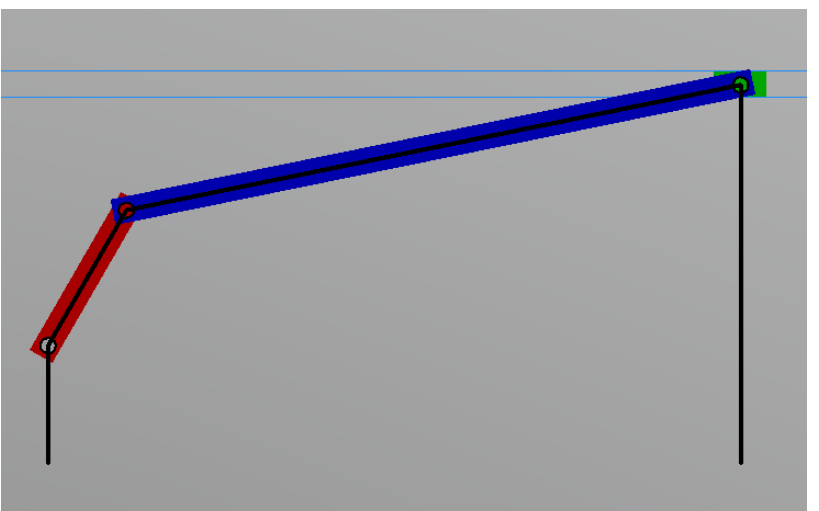

Figura 12 M RRRP descentrado

Fuente: Elaboración Propia

\section{Otros mecanismos simulados}

Otros mecanismos incluidos en las simulaciones, se muestran en las siguientes Figuras.

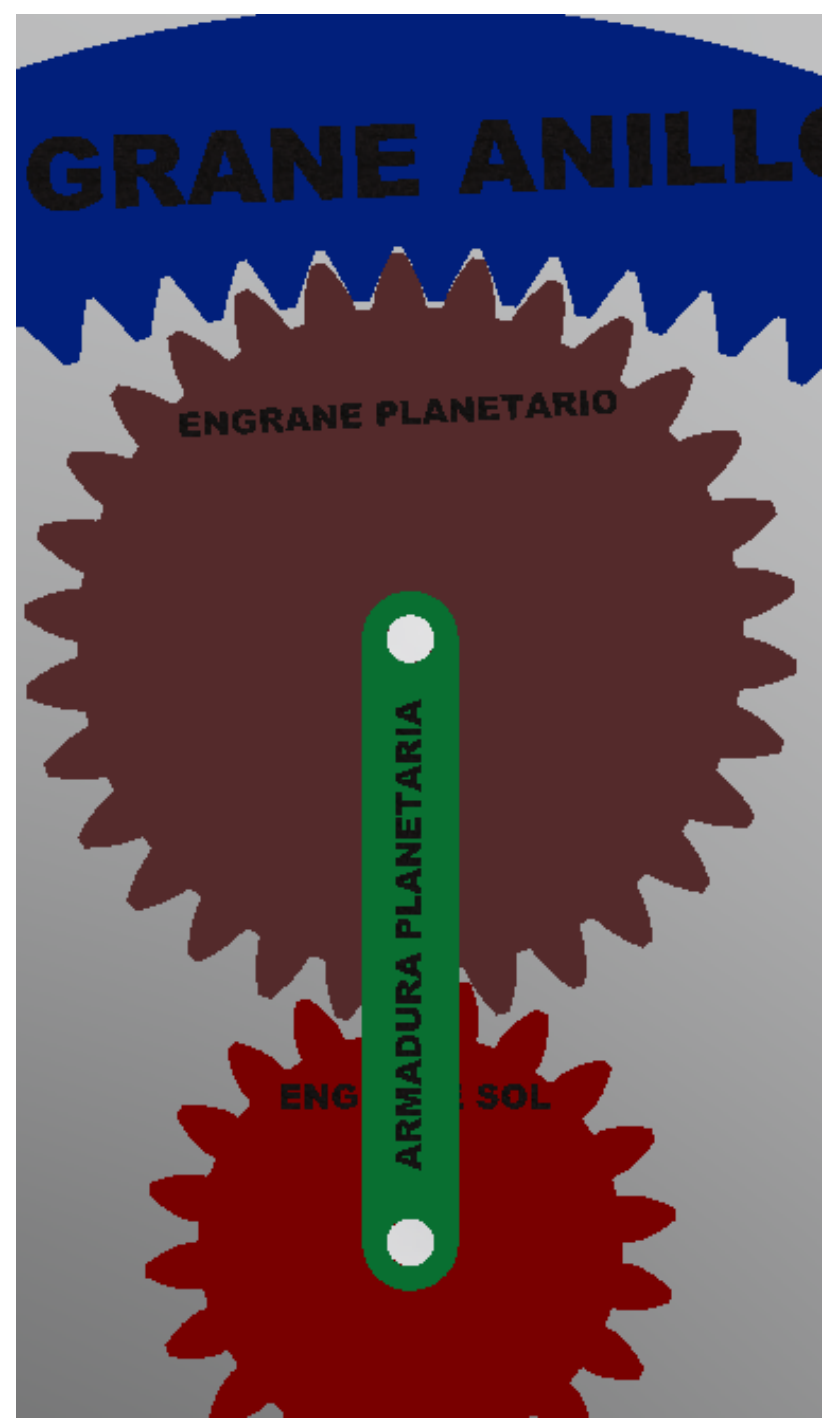

Figura 13 Sistema planetario Fuente: Elaboración Propia

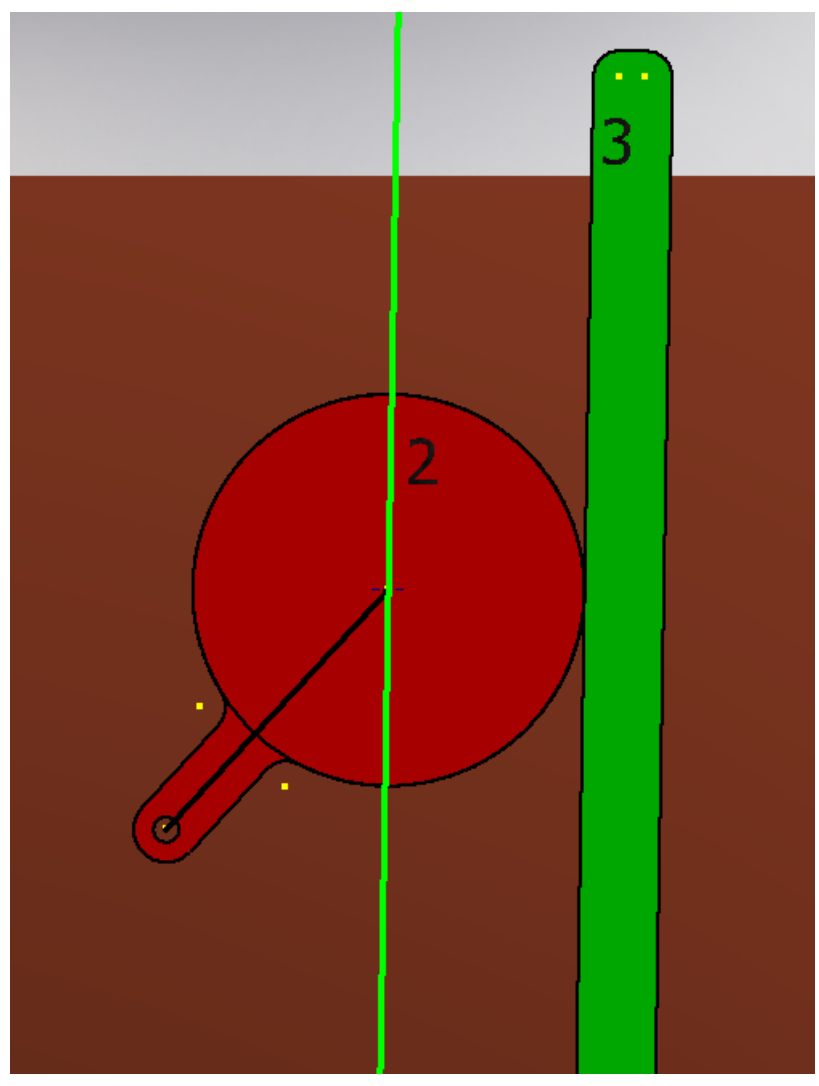

Figura 14 Contacto directo

Fuente: Elaboración Propia

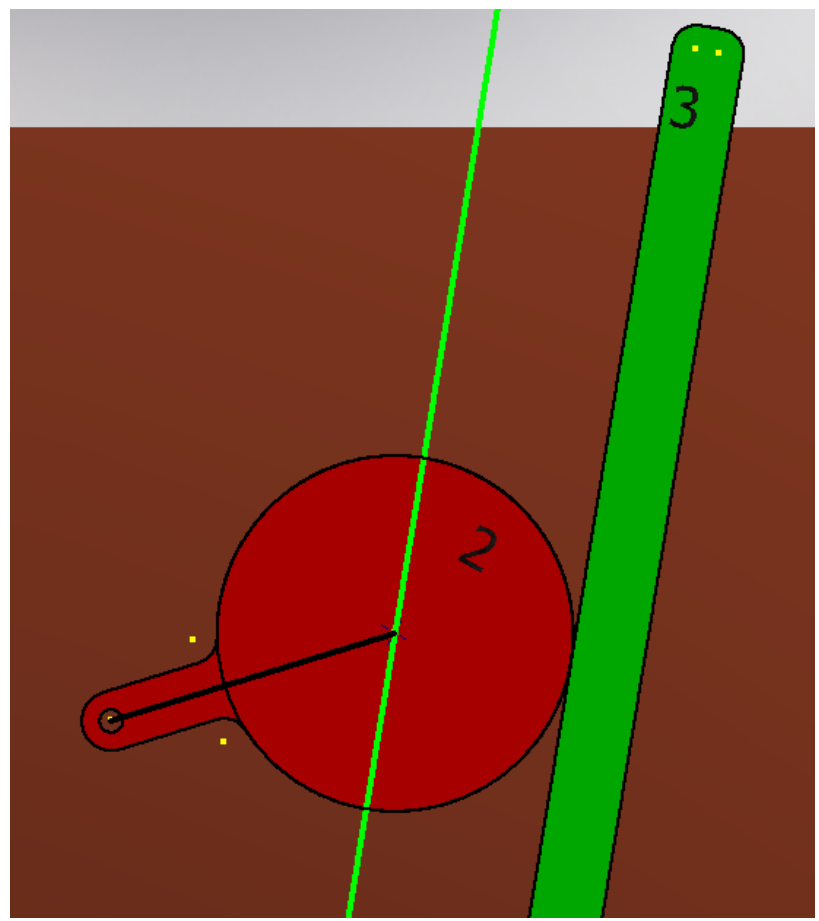

Figura 15 Contacto directo Fuente: Elaboración Propia 


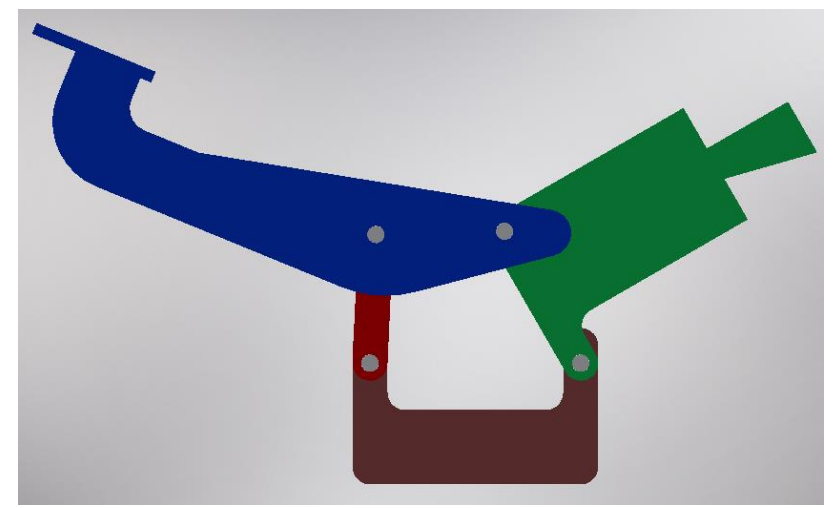

Figura 16 Mecanismo de sujeción Fuente: Elaboración Propia

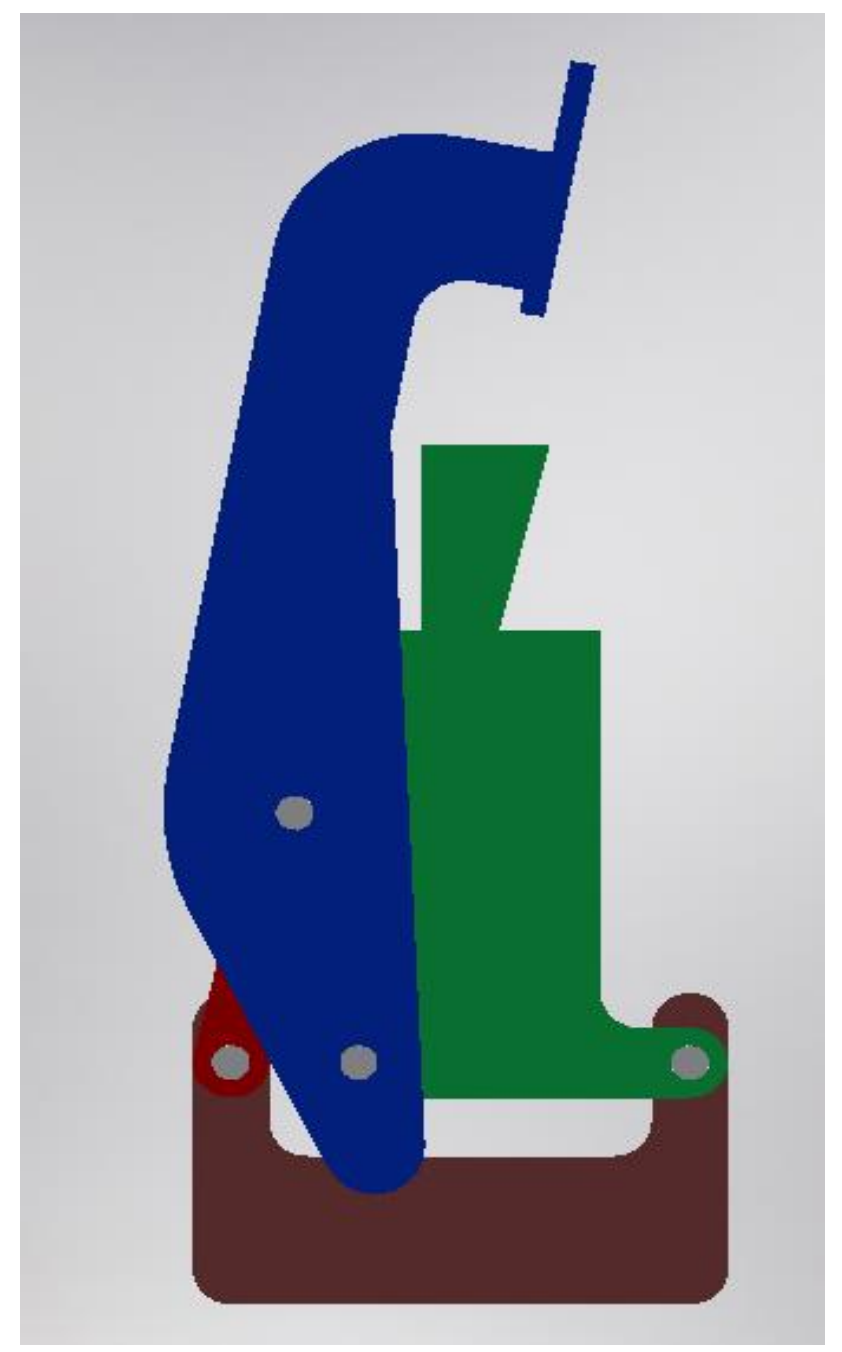

Figura 17 Mecanismo de sujeción

Fuente: Elaboración Propia

\section{Ley de Grashof}

Para comprobar la generalidad del mecanismo de cuatro eslabones RRRR, algunos diagramas cinemáticos de mecanismos RRRR particulares se analizaron a la luz de esta Ley. En la Tabla 2 se muestran datos sobre los correspondientes mecanismos equivalentes; en ella $A=L_{3} C \theta_{3}$ y $B=L_{3} C \beta$.

\begin{tabular}{|l|l|l|c|l|c|}
\hline \multirow{2}{*}{$\mathbf{L}$} & \multicolumn{5}{|c|}{ Figura } \\
& $\mathbf{5}$ & $\mathbf{7}$ & $\mathbf{1 0}$ & $\mathbf{1 1}$ & $\mathbf{1 2}$ \\
\hline $\boldsymbol{L}_{\mathbf{1}}$ & $\boldsymbol{L}_{\mathbf{1}}$ & $\infty+\boldsymbol{A}$ & $\infty$ & $\mathrm{DC}$ & $\infty$ \\
\hline $\boldsymbol{L}_{2}$ & $\boldsymbol{L}_{2}$ & $\infty$ & $\infty$ & $\boldsymbol{r}_{\boldsymbol{b}}$ & $\boldsymbol{L}_{2}$ \\
\hline $\boldsymbol{L}_{3}$ & $\boldsymbol{L}_{\mathbf{1}}$ & $\boldsymbol{L}_{3}$ & $\boldsymbol{L}_{3}$ & $\boldsymbol{L}_{3}$ & $\boldsymbol{L}_{3}$ \\
\hline $\boldsymbol{L}_{\mathbf{4}}$ & $\boldsymbol{L}_{\mathbf{2}}$ & $\infty$ & $\infty+\boldsymbol{B}$ & $\boldsymbol{r}_{\boldsymbol{b}}$ & $\infty+\boldsymbol{e}$ \\
\hline
\end{tabular}

Tabla 2 M RRRR particulares

Fuente: Elaboración Propia

Puesto que en la Fig. 5 son iguales las longitudes de los eslabones 1 y 3, adicionalmente son iguales las longitudes de los eslabones 2 y 4; el mecanismo equivalente será manivela - manivela. En las Figs. 7 y 10 siempre se cumplirá Grashof y los mecanismo son oscilador-oscilador. En la Fig. 12 el mecanismo cumplirá la Ley de Grashof siempre que la diferencia en las longitudes cinemáticas de los eslabones 2 y 3 sea mayor que la excentricidad; si adicionalmente $L_{3}<L_{2}$ el mecanismo será oscilador - oscilador, en caso contrario será manivela - oscilador. Lo aseverado en este párrafo se constata en las simulaciones.

\section{Resultados}

Considerando las longitudes cinemáticas de los eslabones de un mecanismo cualquiera, en función de la ubicación de los centros instantáneos de velocidad del correspondiente mecanismo equivalente RRRR, se constató el cumplimiento de la Ley de Grashof bajo tales condiciones particulares. Adicionalmente, aún cuando no se muestran, los polígonos se pueden obtener para un mecanismo RRRR equivalente con el mismo procedimiento usado para el mecanismo de cuatro barras RRRR.

\section{Agradecimiento}

Los autores agradecemos el apoyo institucional, logístico y financiero de la Universidad Autónoma Metropolitana y de la Unidad azcapotzalco, en particular de la División de Ciencias Básicas e Ingeniería y del Departamento de Energía.

\section{Conclusiones}

Con base en los resultados se concluye que el concepto de mecanismos particulares equivalentes al mecanismo de cuatro barras RRRR, permite el mismo procedimiento para obtener los polígonos correspondientes $\mathrm{y}$, adicionalmente, bajo este enfoque la Ley de Grashof se corrobora en los casos particulares. 


\section{Referencias}

Hartenberg R. S. \& Denavit J.+ (1964). Kinematic Synthesis of Linkages. New York: Mc Graw Hill.

Becker M. C., Salvatore P. \& Zirpoli F. (2005). The impact of virtual simulation tools on problem-solving and new product development organization. ELSEVIER, 34, pp.1305-1321.

Schuler J., Ketchel J. \& Larochelle P... (2007). Computer-Aided Modeling and Manufacturing of Spherical Mechanisms via a novel Web Tool. Journal of Computation, 4, pp. 339-346.

Alvarez J. C. \& Su H. J... (2012). an intuitive virtual environment for supporting the conceptual design of mechanisms. abril 30, 2019, de VRMDS Sitio web: https://doi.org/10.1007/s10055-009-0144-z

López P. R. et al. (2012). Simulación de un Mecanismo Biela-Manivela-Corredera. abril 30, 2019, de Congreso Nacional de Mecatrónica Sitio web: 978-607-95347-7-6

Robson N. \& Ghosh S... (2015). Geometric design of planar mechanisms based on virtual guides for manipulation. abril 30, 2019, de VR Sitio web: https://doi.org/10.1017/S0263574715000272

Caicedo, J. M. G., Reales, R., \& Zambrano, D. (2019). Diseño de la estructura mecánica perteneciente al animatrónico del dinosaurio Laquintasaura. Revista UIS Ingenierías, 18(4), 43-56.

Parra, S. A. G., \& Bedoya, A. E. (2019). Modelo cinemático del martillo de un molino de martillos operando sin carga empleando la mecánica de Lagrange. Ingenierías USBMed, 10(1), 28-33.

Pérez-Rodríguez, R., Adrián-García, Á., \& Simeón-Monet, R. E. (2019). Recuperación del par cinemático pin-buje del cargador frontal Komatsu asistida por computadoras. Ciencias Holguín, 25(1), 47-57.

Rodríguez, Y. E. O., Carbonell, R. A. G., Santiesteban, O. O., Padrón, E. N., \& Hidalgo, Y. A. C. (2019). Análisis cinemático del movimiento de flexión-extensión del dedo pulgar mediante un mecanismo de palanca.
Revista Cubana de Investigaciones Biomédicas, 36(4).

Zevallos, C., \& Irving, D. (2019). Síntesis de un mecanismo de cuatro eslabones para una silla de descanso usando el método de Newton-Raphson. 